\title{
Risk factors for anal high-grade squamous intraepithelial lesions in HIV-positive MSM: is targeted screening possible?
}

\author{
Matthijs L. Siegenbeek van Heukelom ${ }^{a, b, *}$, Elske Marrac,*, \\ Henry J.C. de Vries ${ }^{b, c}$, Maarten F. Schim van der Loeff ${ }^{c}$ \\ and Jan M. Prins ${ }^{\mathrm{a}}$
}

\begin{abstract}
Objective: HIV-positive MSM are at increased risk for developing anal squamous cell carcinoma. Detection of precursor lesions of anal cancer [anal high-grade squamous intraepithelial lesions (HSIL)] is cumbersome and expensive. Our objective was to identify potential risk factors for anal HSIL in HIV-positive MSM to develop more stringent screening criteria.

Design: We studied a cohort of MSM screened by high-resolution anoscopy at three HIV clinics in Amsterdam, the Netherlands.

Methods: For every first high-resolution anoscopy performed in a patient, we analyzed five demographic and seven HIV-related potential risk factors for four different outcome measures: histologically proven anal HSIL vs. no squamous intraepithelial lesions (SIL), HSIL-anal intraepithelial neoplasia 2 vs. no SIL, HSIL-anal intraepithelial neoplasia 3 vs. no SIL, and HSIL vs. no HSIL. We used univariable and multilevel, multivariable logistic regression.
\end{abstract}

Results: From 2008 through 2015, 497 out of 1678 (30\%) screened HIV-positive MSM had anal HSIL. The mean age was 49 years (SD 9.6), 96\% used combination antiretroviral therapy, and median duration of combination antiretroviral therapy use was 7.8 years (interquartile range 4.0-12.4). Increasing age [adjusted odds ratio (aOR) 0.82, $95 \%$ confidence interval $(\mathrm{Cl}) 0.70-0.94, P=0.006$ ] and years living with suppressed viral load [1-5 years suppressed aOR $0.52(95 \% \mathrm{Cl} 0.34-0.80), 5.01-10$ years aOR $0.47(95 \% \mathrm{Cl} 0.29-0.74),>10$ years aOR 0.54 [0.34-0.87], all compared to less than 1 year suppressed, $P=0.009$ ] were found to be protective for HSIL vs. no SIL.

Conclusion: Young HIV-positive MSM without viral suppression are statistically at highest risk for anal HSIL, but given the high prevalence among all virally suppressed men, we advise that all HIV-positive MSM should be screened for HSIL.

Copyright (C 2017 The Author(s). Published by Wolters Kluwer Health, Inc.

AIDS 2017, 31:2295-2301

Keywords: anal intraepithelial neoplasia, HIV, human papillomavirus, risk factor, squamous intraepithelial lesion

\footnotetext{
${ }^{\mathrm{a}}$ Department of Internal Medicine, ${ }^{\mathrm{b}}$ Department of Dermatology, Academic Medical Center, University of Amsterdam, and ${ }^{c}$ Department of Infectious Diseases, Public Health Service Amsterdam, Amsterdam, the Netherlands.

Correspondence to Matthijs L. Siegenbeek van Heukelom, MD, Department of Infectious Diseases, Academic Medical Center, Room F4-106, Meibergdreef 9, 1105 AZ, Amsterdam, the Netherlands.

Tel: +31 20566 6807; fax: +3120 6972286; e-mail: m.l.vanheukelom@amc.uva.nl

* Matthijs L. Siegenbeek van Heukelom and Elske Marra equally contributed to this article.

Received: 26 May 2017; revised: 2 August 2017; accepted: 21 August 2017.
} 


\section{Introduction}

HIV-positive MSM are increasingly at risk for developing anal squamous cell carcinoma compared to the general population [1]. Clinics have been set up to detect precursor lesions of anal squamous cell carcinoma, called squamous intraepithelial lesions (SIL) [2-5]. Highresolution anoscopy (HRA)-guided biopsies are the gold standard for identifying anal SIL. SIL is histopathologically graded anal intraepithelial neoplasia (AIN) 1, 2, or 3 and categorized as low-grade SIL (LSIL; AIN1) or highgrade SIL (HSIL; AIN 2 and 3). The prevalence of histological HSIL (hHSIL) in HIV-positive MSM is around 40\% [6] and, as HRA is a costly and cumbersome procedure, more stringent screening criteria for HIVpositive MSM receiving HRA are needed.

Anal cytology can be used for the detection of HSIL (cytological HSIL), but its use is limited by low sensitivity [7-11]. Several studies have identified risk factors for hHSIL in HIV-positive MSM, but results are not always consistent and studied populations were often relatively small [12-15], limiting their potential to identify the patients at highest risk for hHSIL.

We investigated potential demographic and HIV-related risk factors for the presence of histopathologically proven intra- and perianal HSIL in 1681 HIV-positive MSM screened by HRA in Amsterdam, the Netherlands.

\section{Methods}

\section{Patients and setting}

HIV-positive MSM visiting one of three outpatient HIV clinics in Amsterdam, the Netherlands, were offered AIN screening by their HIV-treating physician. The only exclusion criterion was a life expectancy of less than 12 months. HRA was performed by individually trained anoscopists, did not include anal cytology, sexually transmitted disease (STD), or human papillomavirus (HPV) testing and consisted of a digital rectal examination followed by intra- and perianal inspection with a colposcope (ZEISS opmi pico surgical microscope; Oberkochen, Baden-Württemberg, Germany) after repeatedly applying acetic acid (3-5\% solution) and staining with Lugol's iodine when indicated. Lesions suspicious for SIL were biopsied and graded by pathologists specialized in SIL. In clinics A and C, pathologists used p16 staining for AIN2 graded biopsies, as described by the College of American Pathologists in The Lower Anogenital Squamous Terminology standardization project, whereas, in clinic B, p16 staining was not always used in accordance to the College of American Pathologists Lower Anogenital Squamous Terminology criteria [16]. The highest grade biopsy defined the overall diagnosis.
Data were extracted from the clinical records by two of the investigators (MLSH and EM); behavioral data were patient self-reported to the clinician. HIV-related data were obtained from the Stichting HIV monitoring database [17]. Data collection were performed using the OpenClinica open source software, version 3.6 (OpenClinica LLC and collaborators, Waltham, Massachusetts, USA).

The study (ref. W15_047 \# 15.0058) was approved by the Medical Ethics Review Committee of the Academic Medical Center, Amsterdam, the Netherlands.

\section{Statistical analysis}

Only data of the first HRA of each participant were included. Baseline characteristics of the study population were explored using descriptive statistics, comparing characteristics between participants with no dysplasia, LSIL and HSIL using $\chi^{2}$ test for categorical data and oneway analysis of variance, and Kruskal-Wallis test for continuous data.

Assessed outcome variables were: HSIL vs. no SIL, HSILAIN2 vs. no SIL, HSIL-AIN3 vs. no SIL, and HSIL vs. no HSIL. Five demographic and seven HIV-related variables were included in univariable logistic regression analysis (Suppl. Table 1, http://links.lww.com/QAD/ B156). For $\mathrm{CD}^{+}$cell count and HIV viral load measurements, the measurements closest to the date of HRA were selected. Years living with viral suppression was defined as having a viral load of less than 200 copies/ $\mathrm{ml}$ in tests from August 1999 onwards, and allowing for one blip in viral load below 400 copies/ml between measurements with viral load less than 200 copies $/ \mathrm{ml}$ [18]. For samples tested prior to August 1999 the cut-off of detectability of the laboratory assay was the cut-off used to define viral suppression.

Multivariable logistic regression analysis was used to determine independent risk factors for HSIL among HIV-positive MSM. All variables that were significantly associated in univariable logistic regression analysis with HSIL diagnosis (at $P<0.2$, Wald test) were included in the multivariable analysis, as well as the following a priori selected parameters (risk factors for HSIL in other studies [10,19-22]): age at time of HRA, a history of at least one AIDS defining illness, nadir $\mathrm{CD}^{+}{ }^{+}$cell count, current $\mathrm{CD}^{+}{ }^{+}$cell count, and years living with viral suppression. A backward selection method was used to create a parsimonious model in which a priori selected variables were forced. We conducted multilevel multivariable logistic regression analyses to correct for potential similarities of participants within each clinic. Multicollinearity was tested with the variance inflation factor and if present, the most objective measurable variable remained in the model, whereas the other variable was dropped. However, the effect of this exclusion was checked in a sensitivity analysis. To assess whether the 
effect of risk factors differed by clinic, interaction was tested in the multivariable model. Also, risk factors for HSIL were assessed separately for intra-anal and perianal HSIL. Variables were considered significantly associated when $P$ value was less than 0.05 . Statistical analyses were performed using Stata (version 13.1; Stata Corp, College Station, Texas, USA).

\section{Results}

\section{Characteristics of study population}

Between 12 February 2008 and 24 November 2015, 1681 HIV-positive MSM underwent their first HRA. Potential risk factors for histological (H)SIL of $311 \mathrm{HIV-positive}$ MSM have been previously described [15]. Three participants were excluded based on the diagnosis of SCC (two intra-anal and one perianal SCC). The mean age was 49 years (SD 9.6) and $96 \%$ of participants used combination antiretroviral therapy (cART). Median duration of cART use was 7.8 years [interquartile range (IQR) 4.0-12.4], median nadir CD $4^{+}$cell count was 220 cells/ $\mu$ l (IQR 130-320), and median current $\mathrm{CD} 4^{+}$cell count was 620 cells/ $\mu$ l (IQR 480-790). Only 354 (22\%) participants had at least one AIDS-defining illness prior to HRA (Table 1). There were some statistically significant (but clinically not important) differences between patients from the three clinics (Suppl. Table 2, http://links.lww. com/QAD/B156).
Of the 1678 HIV-positive MSM, 396 (24\%) were diagnosed with AIN1, 289 (17\%) with AIN2, and 208 with AIN3 (12\%). The prevalence of HSIL was $30 \%$ (497/1678 participants). The prevalence of HSIL did not differ significantly between clinics (Suppl. Table 2, http://links.lww.com/QAD/B156). Compared to participants with no dysplasia, participants with LSIL and HSIL were younger $(P<0.001)$, had more sex partners in the preceding 6 months $(P=0.016)$, had a lower number of years on cART $(P<0.001)$, had a different distribution of nadir $\mathrm{CD} 4^{+}$cell counts $(P=0.005)$, had more often a detectable HIV viral load $(P=0.001)$, and had less years living with viral suppression $(P<0.001$; Table 1$)$.

\section{Univariable logistic regression analyses}

Associated variables for HSIL vs. no SIL in univariable logistic regression analyses were age, duration of cART use, having had an STD in the preceding 6 months, HIV plasma viral load, and number of years living with viral suppression. Nadir and current $\mathrm{CD} 4{ }^{+}$cell count were not associated with HSIL. Univariable associations between risk factors and outcome were largely similar between the four outcome measures (Suppl. Table 1, http:// links.lww.com/QAD/B156).

\section{Multivariable logistic regression analyses}

In multivariable logistic regression analysis, nested within clinic and comparing HSIL vs. no SIL, increasing age [adjusted odds ratio (aOR) 0.82, 95\% confidence interval

Table 1. Characteristics of the study population of the anal intraepithelial neoplasia cohort study by histological high-grade squamous intraepithelial lesion status, Amsterdam 2008-2015 $(N=1678)$.

\begin{tabular}{|c|c|c|c|c|c|c|c|c|c|}
\hline & \multicolumn{2}{|c|}{$\begin{array}{c}\text { Total } \\
(N=1678)\end{array}$} & \multicolumn{2}{|c|}{$\begin{array}{l}\text { No dysplasia } \\
(N=785)\end{array}$} & \multicolumn{2}{|c|}{$\begin{array}{c}\text { LSIL } \\
(N=396)\end{array}$} & \multicolumn{2}{|c|}{$\begin{array}{c}\text { HSIL } \\
(N=497)\end{array}$} & \multirow[b]{2}{*}{$P$ value } \\
\hline & No. & $\%$ & No & $\%$ & No. & $\%$ & No. & $\%$ & \\
\hline \multicolumn{10}{|l|}{ Demographic variables } \\
\hline Age in years $(\text { mean/SD) })^{a}$ & 49 & (9.6) & 50 & (9.4) & 48 & $(10.0)$ & 48 & $(9.3)$ & $<0.001$ \\
\hline Continent of birth ${ }^{b}$ & & & & & & & & & 0.710 \\
\hline Europe & 1279 & $82 \%$ & 566 & $82 \%$ & 305 & $81 \%$ & 408 & $84 \%$ & \\
\hline Americas & 188 & $12 \%$ & 83 & $12 \%$ & 50 & $13 \%$ & 55 & $11 \%$ & \\
\hline Oceania & 11 & $1 \%$ & 5 & $1 \%$ & 1 & $0 \%$ & 5 & $1 \%$ & \\
\hline Asia & 46 & $3 \%$ & 22 & $3 \%$ & 14 & $4 \%$ & 10 & $2 \%$ & \\
\hline Africa & 28 & $2 \%$ & 13 & $2 \%$ & 8 & $2 \%$ & 7 & $1 \%$ & \\
\hline Smoking status ${ }^{\mathrm{C}}$ & & & & & & & & & 0.481 \\
\hline Never smoked & 598 & $38 \%$ & 275 & $38 \%$ & 145 & $39 \%$ & 178 & $39 \%$ & \\
\hline Previously smoking & 379 & $24 \%$ & 191 & $26 \%$ & 83 & $22 \%$ & 105 & $23 \%$ & \\
\hline Currently smoking & 577 & $37 \%$ & 256 & $35 \%$ & 145 & $39 \%$ & 176 & $38 \%$ & \\
\hline $\begin{array}{l}\text { Number of sex partners in the preceding } \\
6 \text { months (median/lQR) }\end{array}$ & 2 & $(1-6)$ & 1 & $(1-5)$ & 2 & $(1-6)$ & 2 & $(1-7)$ & 0.016 \\
\hline Number of sex partners in the preceding 6 months ${ }^{\mathrm{d}}$ & & & & & & & & & 0.166 \\
\hline $0-1$ & 598 & $49 \%$ & 295 & $52 \%$ & 141 & $48 \%$ & 162 & $44 \%$ & \\
\hline $2-5$ & 304 & $25 \%$ & 131 & $23 \%$ & 77 & $26 \%$ & 96 & $26 \%$ & \\
\hline$\geq 6$ & 316 & $26 \%$ & 136 & $24 \%$ & 73 & $25 \%$ & 107 & $29 \%$ & \\
\hline Had an STD in the preceding 6 months & & & & & & & & & 0.066 \\
\hline No & 1586 & $95 \%$ & 750 & $96 \%$ & 376 & $95 \%$ & 460 & $93 \%$ & \\
\hline Yes & 92 & $5 \%$ & 35 & $4 \%$ & 20 & $5 \%$ & 37 & $7 \%$ & \\
\hline \multicolumn{10}{|l|}{ HIV-related variables } \\
\hline Currently using $\mathrm{CART}^{\mathrm{e}}$ & & & & & & & & & 0.124 \\
\hline No & 73 & $4 \%$ & 26 & $3 \%$ & 23 & $6 \%$ & 24 & $5 \%$ & \\
\hline Yes & 1580 & $96 \%$ & 743 & $97 \%$ & 368 & $94 \%$ & 469 & $95 \%$ & \\
\hline Duration of cART use in years (median/lQR) ${ }^{f}$ & 7.8 & $(4.0-12.4)$ & 8.9 & $(4.8-12.8)$ & 6.6 & $(3.6-11.8)$ & 7.5 & $(3.1-12.3)$ & $<0.001$ \\
\hline
\end{tabular}


Table 1 (continued)

\begin{tabular}{|c|c|c|c|c|c|c|c|c|c|}
\hline & \multicolumn{2}{|c|}{$\begin{array}{c}\text { Total } \\
(N=1678)\end{array}$} & \multicolumn{2}{|c|}{$\begin{array}{c}\text { No dysplasia } \\
\quad(N=785)\end{array}$} & \multicolumn{2}{|c|}{$\begin{array}{c}\text { LSIL } \\
(N=396)\end{array}$} & \multicolumn{2}{|c|}{$\begin{array}{c}\text { HSIL } \\
(N=497)\end{array}$} & \multirow[b]{2}{*}{$P$ value } \\
\hline & No. & $\%$ & No & $\%$ & No. & $\%$ & No. & $\%$ & \\
\hline $\mathrm{CD}^{+}{ }^{+} \mathrm{T}$-cell count cells/ $\mu \mathrm{l}\left(\right.$ median/lQR) ${ }^{\mathrm{g}}$ & 620 & $(480-790)$ & 620 & $(480-800)$ & 605 & $(450-780)$ & 630 & $(490-800)$ & 0.236 \\
\hline $\mathrm{CD}^{+}{ }^{\mathrm{T}}$-cell count cells/$/\left.\mu\right|^{\mathrm{g}}$ & & & & & & & & & 0.423 \\
\hline$<350$ & 168 & $10 \%$ & 75 & $10 \%$ & 48 & $12 \%$ & 45 & $9 \%$ & \\
\hline $350-500$ & 323 & $20 \%$ & 153 & $20 \%$ & 81 & $21 \%$ & 89 & $18 \%$ & \\
\hline$>500$ & 1163 & $70 \%$ & 541 & $70 \%$ & 265 & $67 \%$ & 357 & $73 \%$ & \\
\hline Nadir CD4 ${ }^{+}$T-cell count cells/ $\mu$ I (median/lQR) ${ }^{\mathrm{h}}$ & 220 & $(130-320)$ & 220 & $(130-310)$ & 220 & $(120-320)$ & 222 & $(130-351)$ & 0.357 \\
\hline Nadir CD4 ${ }^{+}$T-cell count cells $/\left.\mu\right|^{\mathrm{h}}$ & & & & & & & & & 0.005 \\
\hline$<100$ & 315 & $19 \%$ & 146 & $19 \%$ & 79 & $20 \%$ & 90 & $18 \%$ & \\
\hline $100-199$ & 368 & $22 \%$ & 168 & $22 \%$ & 86 & $22 \%$ & 114 & $23 \%$ & \\
\hline $200-349$ & 624 & $38 \%$ & 315 & $41 \%$ & 153 & $39 \%$ & 156 & $32 \%$ & \\
\hline$>350$ & 344 & $21 \%$ & 138 & $18 \%$ & 76 & $19 \%$ & 130 & $26 \%$ & \\
\hline HIV plasma viral load copies/ml (median/IQR) ${ }^{\mathrm{i}}$ & 20 & $(1-40)$ & 20 & $(1-40)$ & 20 & $(1-40)$ & 20 & $(1-40)$ & 0.211 \\
\hline HIV viral load copies/mi & & & & & & & & & 0.001 \\
\hline$<50$ & 1347 & $89 \%$ & 620 & $92 \%$ & 315 & $85 \%$ & 412 & $88 \%$ & \\
\hline$>50$ & 161 & $11 \%$ & 51 & $8 \%$ & 56 & $15 \%$ & 54 & $12 \%$ & \\
\hline Years living with viral suppression (median/IQR) & 6.3 & $(2.6-11.1)$ & 7.2 & $(3.7-11.8)$ & 5.1 & $(2.0-10.1)$ & 5.9 & $(1.9-11.0)$ & $<0.001$ \\
\hline Years living with viral suppression, ${ }^{\mathrm{j}, \mathrm{k}}$ & & & & & & & & & $<0.001$ \\
\hline$<1$ year & 203 & $14 \%$ & 61 & $9 \%$ & 57 & $16 \%$ & 85 & $18 \%$ & \\
\hline $1-5$ years & 411 & $28 \%$ & 172 & $26 \%$ & 117 & $33 \%$ & 122 & $26 \%$ & \\
\hline $5.01-10$ years & 393 & $27 \%$ & 187 & $28 \%$ & 94 & $26 \%$ & 112 & $24 \%$ & \\
\hline$>10$ years & 476 & $32 \%$ & 238 & $36 \%$ & 92 & $26 \%$ & 146 & $31 \%$ & \\
\hline Having had an AIDS defining illness ${ }^{m, n}$ & & & & & & & & & 0.803 \\
\hline No & 1280 & $78 \%$ & 575 & $78 \%$ & 313 & $79 \%$ & 392 & $79 \%$ & \\
\hline Yes & 354 & $22 \%$ & 166 & $22 \%$ & 83 & $21 \%$ & 105 & $21 \%$ & \\
\hline Clinic & & & & & & & & & \\
\hline Clinic where HRA was done & & & & & & & & & 0.008 \\
\hline Clinic A & 710 & $42 \%$ & 334 & $43 \%$ & 163 & $41 \%$ & 213 & $43 \%$ & \\
\hline Clinic B & 674 & $40 \%$ & 288 & $37 \%$ & 176 & $44 \%$ & 210 & $42 \%$ & \\
\hline Clinic C & 294 & $17 \%$ & 163 & $21 \%$ & 57 & $14 \%$ & 74 & $15 \%$ & \\
\hline AIN diagnosis & & & & & & & & & \\
\hline No dysplasia & 785 & $47 \%$ & & & & & & & \\
\hline AIN1 & 396 & $24 \%$ & & & & & & & \\
\hline AIN2 & 289 & $17 \%$ & & & & & & & \\
\hline AIN3 & 208 & $12 \%$ & & & & & & & \\
\hline Location HSIL & & & & & & & & & \\
\hline Intra-anal HSIL & 474 & $28 \%$ & & & & & & & \\
\hline Perianal HSIL & 46 & $3 \%$ & & & & & & & \\
\hline
\end{tabular}

AIN, anal intraepithelial neoplasia; cART, combination antiretroviral therapy; HSIL, high-grade squamous intraepithelial lesion; IQR, interquartile range; LSIL, low-grade squamous intraepithelial lesion; STD, sexually transmitted disease.

${ }^{\text {aTTtal }}$ - 1 missing; no dysplasia - 1 missing; LSIL - 0 missings; HSIL - 0 missings.

${ }^{\mathrm{b}}$ Total - 126 missings; no dysplasia - 96 missings; LSIL - 18 missings; HSIL - 12 missings.

'Total - 124 missings; no dysplasia - 63 missings; LSIL - 23 missings; HSIL - 38 missings.

${ }^{\mathrm{d}}$ Total - 460 missings; no dysplasia - 223 missings; LSIL - 105 missings; HSIL - 132 missings.

${ }^{\mathrm{e} T o t a l}$ - 25 missings; no dysplasia - 16 missings; LSIL - 5 missings; HSIL - 4 missings.

'Total - 93 missings; no dysplasia - 39 missings; LSIL - 27 missings; HSIL - 27 missings.

gTotal - 24 missings; no dysplasia - 16 missings; LSIL - 2 missings; HSIL -6 missings.

hTotal - 27 misisngs; no dysplasia - 18 missings; LSIL - 2 missings; HSIL -7 missings.

'Total - 170 missings; no dysplasia - 113 missings; LSIL - 26 missings; HSIL - 31 missings.

'Total - 196 missings; no dysplasia - 127 missings; LSIL - 36 missings; HSIL - 33 missings.

${ }^{k}$ Viral suppression was defined as having a viral load of less than 200 in tests from 1 August 1999 onwards allowing for a onetime blip in viral load between 200 and 400 copies/ml. For samples tested prior to 1 August 1999 the cut-off of detectability of the laboratory assay that was used for that sample is the cut-off for viral suppression.

IParticipants who never had an undetectable viral load are included in the category less than 1-year undetectable viral load.

${ }^{\mathrm{m}}$ Total - 45 missings; no dysplasia - 44 missings; LSIL - 0 missing; HSIL -1 missing.

nIncluded AIDS defining illnesses: candidiasis esophageal, Kaposi's sarcoma, recurrent pneumonia, chronic intestinal microsporidiosis, pneumocystis carinii pneumonia, Mycobacterium kansasii, pulmonary tuberculosis, chronic intestinal cryptosporidiosis, AIDS dementia complex/HIV encephalopathy, toxoplasmosis of the brain, other species/unidentified extrapulmonary mycobacterium, cytomegalovirus (CMV) retinitis, nonHodgkin's lymphoma, CMV disease (other than lymph node, liver, spleen), herpes simplex virus, progressive multifocal leucoencephalopathy, extrapulmonary cryptococcosis, primary lymphoma of central nervous system, extrapulmonary tuberculosis, wasting syndrome because of HIV, disseminated or extrapulmonary histoplasmosis, visceral leishmaniasis, candidiasis of trachea/bronchi/lungs, disseminated or extrapulmonary coccidioidomycosis, extrapulmonary pneumocystis, other CDC C event. 
Table 2. Risk factors of anal histologically proven high-grade squamous intraepithelial lesion.

\begin{tabular}{|c|c|c|c|c|c|c|c|c|c|c|c|c|}
\hline & \multicolumn{3}{|c|}{$\begin{array}{l}\text { (1) Multivariable } \\
\text { logistic regression } \\
\text { HSIL vs. no SIL }\end{array}$} & \multicolumn{3}{|c|}{$\begin{array}{l}\text { (2) Multivariable } \\
\text { logistic regression } \\
\text { AIN2 vs. no SIL }\end{array}$} & \multicolumn{3}{|c|}{$\begin{array}{l}\text { (3) Multivariable } \\
\text { logistic regression } \\
\text { AIN3 vs. no SIL }\end{array}$} & \multicolumn{3}{|c|}{$\begin{array}{l}\text { (4) Multivariable } \\
\text { logistic regression } \\
\text { HSIL vs. no HSIL }\end{array}$} \\
\hline & $\mathrm{aOR}$ & $(95 \% \mathrm{Cl})$ & $P$ & $\mathrm{aOR}$ & $(95 \% \mathrm{Cl})$ & $P$ & $\mathrm{aOR}$ & $(95 \% \mathrm{Cl})$ & $P$ & $\mathrm{aOR}$ & $(95 \% \mathrm{Cl})$ & $P$ \\
\hline \multicolumn{13}{|l|}{ Demographic variables } \\
\hline Age in years ${ }^{b}$ & 0.82 & $(0.70-0.94)$ & 0.006 & 0.79 & $(0.66-0.93)$ & 0.006 & 0.85 & $(0.70-1.04)$ & 0.116 & 0.88 & $(0.77-1.00)$ & 0.057 \\
\hline \multicolumn{13}{|l|}{ HIV-related variables } \\
\hline $\mathrm{CD}^{+}{ }^{-}$-cell count, cells/ $\mu \mathrm{l}$ & & & 0.427 & & & 0.757 & & & 0.290 & & & 0.397 \\
\hline$<350$ & REF & & & REF & & & REF & & & REF & & \\
\hline $350-500$ & 1.08 & $(0.64-1.82)$ & & 1.05 & $(0.56-1.95)$ & & 1.13 & $(0.55-2.30)$ & & 1.15 & $(0.72-1.84)$ & \\
\hline$>500$ & 1.29 & $(0.79-2.10)$ & & 1.18 & $(0.66-2.13)$ & & 1.50 & $(0.77-2.91)$ & & 1.31 & $(0.84-2.04)$ & \\
\hline Nadir CD4 T-cell count, cells $/ \mu \mathrm{I}^{\mathrm{C}}$ & 0.97 & $(0.88-1.06)$ & 0.494 & 1.02 & $(0.92-1.14)$ & 0.671 & 0.89 & $(0.78-1.01)$ & 0.076 & 1.00 & $(0.92-1.09)$ & 0.924 \\
\hline Years living with viral suppression ${ }^{\mathrm{d}}$ & & & 0.009 & & & 0.070 & & & 0.006 & & & 0.039 \\
\hline$<1$ year $^{\mathrm{e}}$ & REF & & & REF & & & REF & & & REF & & \\
\hline $1-5$ years & 0.52 & $(0.34-0.80)$ & & 0.53 & $(0.32-0.86)$ & & 0.49 & $(0.28-0.85)$ & & 0.61 & $(0.42-0.88)$ & \\
\hline $5.01-10$ years & 0.47 & $(0.29-0.74)$ & & 0.55 & $(0.32-0.94)$ & & 0.34 & $(0.19-0.64)$ & & 0.61 & $(0.41-0.92)$ & \\
\hline$>10$ years & 0.54 & $(0.34-0.87)$ & & 0.54 & $(0.31-0.93)$ & & 0.51 & $(0.28-0.94)$ & & 0.73 & $(0.48-1.11)$ & \\
\hline Having had an AIDS defining illness & & & 0.899 & & & 0.777 & & & 0.888 & & & 0.995 \\
\hline No & REF & & & REF & & & REF & & & REF & & \\
\hline Yes & 0.98 & $(0.73-1.32)$ & & 0.95 & $(0.66-1.37)$ & & 1.03 & $(0.69-1.53)$ & & 1.00 & $(0.76-1.32)$ & \\
\hline
\end{tabular}

(1) High-grade squamous intraepithelial lesion vs. no squamous intraepithelial lesion; (2) Anal intraepithelial neoplasia 2 vs. no squamous intraepithelial lesion; (3) Anal intraepithelial neoplasia 3 vs. no squamous intraepithelial lesion; (4) High-grade squamous intraepithelial lesion vs. no high-grade squamous intraepithelial lesion (including anal intraepithelial neoplasia 1) ${ }^{f}$. Cl, confidence interval; HRA, high-resolution anoscopy; HSIL, high-grade squamous intraepithelial lesion; IQR, interquartile range; SIL, squamous intraepithelial lesion; STD, sexually transmitted disease. ${ }^{a}$ Multivariable model HSIL vs. no SIL includes 1120 participants; multivariable model AIN2 vs. no SIL includes 929 participants; multivariable model AIN3 vs. no SIL includes 847 participants; multivariable model HSIL vs. no HSIL includes 1480 participants.

${ }^{\mathrm{b}}$ Per 10-year increase in age.

${ }^{\mathrm{c}}$ Per $100 \mathrm{cells} / \mu \mathrm{l}$ increase.

${ }^{\mathrm{d} V i r a l}$ suppression was defined as having a viral load of less than 200 in tests from 1 August 1999 onwards allowing for a onetime blip in viral load between 200 and 400 copies $/ \mathrm{ml}$. For samples tested prior to 1 August 1999 the cut-off of detectability of the laboratory assay that was used for that sample is the cut-off for viral suppression. This varies by time period (sensitivity of the assays increased over time) and hospital (based on the used assay).

eParticipants who never had an undetectable viral load are included in the category less than 1-year undetectable viral load.

IIndividuals were nested within clinic where HRA was done (multilevel analysis).

(CI) $0.70-0.94 ; P=0.006]$, and years living with suppressed viral load were significantly protective for HSIL [1-5 years viral suppression aOR $0.52(95 \% \mathrm{CI}$ $0.34-0.80), 5.01-10$ years viral suppression aOR 0.47 (95\% CI $0.29-0.74),>10$ years viral suppression aOR 0.54 (0.34-0.87), all compared to less than 1 year viral suppression, $P=0.009]$. Outcome measures HSIL-AIN2 vs. no SIL, HSIL-AIN3 vs. no SIL, and HSIL vs. no HSIL yielded similar results (Table 2).

Based on multicollinearity, we excluded a priori cART use, HIV viral load and duration of cART use because this is largely represented in number of years living with viral suppression. We reran the model including duration of cART use instead of years living with viral suppression, yielding similar results. None of the interaction terms were significant, suggesting that the effect of the included risk factors did not vary by clinic (data not shown).

Additionally, we assessed risk factors for intra- and perianal HSIL vs. no SIL separately. Intra-anal HSIL showed results comparable to the overall model. Only being a current smoker was found to be a significant risk factor of perianal HSIL in multilevel multivariable logistic regression analyses [previously smoked aOR 2.41 (95\% CI 0.77-7.57), current smoker aOR 4.90 (95\% CI 1.8013.35), compared to participants who never smoked,
$P=0.006$; Supplementary Table 3, http://links.lww.com/ $\mathrm{QAD} / \mathrm{B} 156]$.

\section{Discussion}

We analyzed potential risk factors for anal HSIL (AIN2-3) in 1678 HIV-positive MSM. Of five demographic and seven HIV-related potential risk factors, only increasing age and years living with suppressed viral load were significantly protective for HSIL vs. no SIL. Sensitivity analyses, including duration of cART use instead of duration of viral suppression, showed a similar relation.

In contrast to our results, several smaller studies, with less than 400 patients each, reported various demographic and HIV-related risk factors that significantly increased the risk for anal hHSIL: the number of specific HPV types and current use of cART [13], increasing age and CD4 ${ }^{+}$ cell counts less than $50 / \mu l$ before starting cART [12], and smoking $[23,24]$. We found smoking to be a risk factor, but for perianal HSIL only. We also confirmed that duration of cART use showed a reduced risk for hHSIL $[12,25]$. Living more than 1 year with viral suppression might reduce the risk for hHSIL as it could be a proxy for immune restoration over time. We have no good explanation how older age could be protective for anal HSIL. 
The strength of this study is that it reports nearly 1700 patients from three HIV clinics, using hHSIL as an endpoint. This allowed for an extensive multivariable analysis without constraints in the number of covariates.

A limitation of our study is that no data were collected on anal HPV infections, but given that 95\% of all HIV-positive MSM have anal HPV present, the value of this risk factor is debatable $[13,26]$. More specifically, HPV16 testing might be considered to have more discriminatory power, but given that HPV16 was the causative HPV type in only $60 \%$ of anal carcinomas in HIV-positive MSM in another study by our group [27], 40\% of potential anal carcinomas would be missed. Also, patient populations differed significantly between clinics, which may be explained by differences in calendar year of starting with HRA screening and by the large study populations, easily leading to statistically significant but not clinically important differences. Furthermore, multiple anoscopists performed the HRAs, and given the long learning curve for HRA, some HSIL lesions may have been missed $[28,29]$. Also, in clinic B p16 staining was not always used to confirm AIN2 graded biopsies. Finally, social desirability bias might have occurred for self-reported STDs and sexual behavior.

Young HIV-positive MSM without viral suppression are statistically at highest risk for HSIL, but given the high prevalence of HSIL among all virally suppressed men, we advise that all HIV-positive MSM should be screened for the presence of anal HSIL.

\section{Acknowledgements}

We thank Michiel Nieuwenhuis and Djurre Bismijer for data collection and the Stichting HIV Monitoring (SHM) for sharing data on HIV-related parameters.

M.S.V.D.L. reports grants from Merck, Sanofi Pasteur MSD, and Janssen outside the submitted work. H.J.C.D.V. reports a grant from Medicine outside the submitted work.

The abstract of this manuscript has been presented at the International Anal Neoplasia Society Scientific Meeting in San Francisco, California, USA, 11-13 November 2016.

\section{Conflicts of interest}

There are no conflicts of interest.

\section{References}

1. Hleyhel $M$, Hleyhel $M$, Bouvier AM, Belot $A$, Tattevin $P$ Pacanowski J, et al., Cancer Risk Group of the French Hospital Database on HIV (FHDH-ANRS CO4). Risk of non-AIDS-defining cancers among HIV-1-infected individuals in France between 1997 and 2009: results from a French cohort. AIDS 2014; 28:2109-2118.

2. Jay N. Elements of an anal dysplasia screening program. J Assoc Nurses AIDS Care 2011; 22:465-477.
3. Kreuter A, Potthoff A, Brockmeyer NH, Gambichler T, Swoboda J, Stucker M, et al., German Competence Network HIV/AIDS. Anal carcinoma in human immunodeficiency virus-positive men: results of a prospective study from Germany. $\mathrm{Br} / \mathrm{Der}$ matol 2010; 162:1269-1277.

4. Park IU, Palefsky JM. Evaluation and management of anal intraepithelial neoplasia in HIV-negative and HIV-positive men who have sex with men. Curr Infect Dis Rep 2010; 12:126-133.

5. Watson AJ, Smith BB, Whitehead MR, Sykes PH, Frizelle FA. Malignant progression of anal intra-epithelial neoplasia. ANZ J Surg 2006; 76:715-717.

6. Machalek DA, Poynten M, Jin F, Fairley CK, Farnsworth A, Garland $\mathrm{SM}$, et al. Anal human papillomavirus infection and associated neoplastic lesions in men who have sex with men: a systematic review and meta-analysis. Lancet Oncol 2012; 13:487-500.

7. Nathan M, Singh N, Garrett N, Hickey N, Prevost T, Sheaff M. Performance of anal cytology in a clinical setting when measured against histology and high-resolution anoscopy findings. AIDS 2010; 24:373-379.

8. Hillman RJ, Gunathilake MP, Jin F, Tong W, Field A, Carr A. Ability to detect high-grade squamous anal intraepithelial lesions at high resolution anoscopy improves over time. Sex Health 2016: 13:177-181.

9. Berry JM, Palefsky JM, Jay N, Cheng SC, Darragh TM, ChinHong PV. Performance characteristics of anal cytology and human papillomavirus testing in patients with high-resolution anoscopy-guided biopsy of high-grade anal intraepithelial neoplasia. Dis Colon Rectum 2009; 52:239-247.

10. D'Souza G, Wentz A, Wiley D, Shah N, Barrington F, Darragh $\mathrm{TM}$, et al. Anal cancer screening in men who have sex with men in the multicenter AIDS cohort study. I Acquir Immune Defic Syndr 2016; 71:570-576.

11. Darragh TM, Winkler B. Anal cancer and cervical cancer screening: key differences. Cancer Cytopathol 2011; 119:5-19.

12. de Pokomandy A, Rouleau D, Ghattas G, Trottier H, Vezina S, Cote $\mathrm{P}$, et al. HAART and progression to high-grade anal intraepithelial neoplasia in men who have sex with men and are infected with HIV. Clin Infect Dis 2011; 52:1174-1181.

13. Palefsky JM, Holly EA, Efirdc JT, Da Costa M, Jay N, Berry JM, Darragh TM. Anal intraepithelial neoplasia in the highly active antiretroviral therapy era among HIV-positive men who have sex with men. AIDS 2005; 19:1407-1414.

14. van der Snoek EM, van der Ende ME, den Hollander IC, Schutten $M$, Neumann HA, van Doornum GJ. Use of highly active antiretroviral therapy is associated with lower prevalence of anal intraepithelial neoplastic lesions and lower prevalence of human papillomavirus in HIV-infected men who have sex with men. Sex Transm Dis 2012; 39:495-500.

15. Richel O, De Vries HJ, Dijkgraaf MG, Van Noesel CJ, Prins JM. Risk factors for the presence of anal intraepithelial neoplasia in HIV+ men who have sex with men. PLoS One 2013; 8:e84030.

16. Darragh TM, Colgan TJ, Cox JT, Heller DS, Henry MR, Luff RD, et al., Members of LAST Project Work Groups. The Lower anogenital squamous terminology standardization project for HPV-associated lesions: background and consensus recommendations from the college of American pathologists and the American society for colposcopy and cervical pathology. J Low Genit Tract Dis 2012; 16:205-242.

17. Stichting HIV Monitoring. Available at https://www.hiv-monitoring.nl/english. [Accessed 8 February 2017]

18. Panel on Antiretroviral Guidelines for Adults and Adolescents. Department of Health and Human Services. Guidelines for the use of antiretroviral agents in HIV-1-infected adults and adolescents. Available at http://aidsinfo.nih.gov/contentfiles/lvguidelines/AdultandAdolescentGL.pdf. [Accessed 4 November 2017]

19. Conley L, Bush T, Darragh TM, Palefsky JM, Unger ER, Patel P, et al., Study to Understand the Natural History of HIV and AIDS in the Era of Effective Therapy (SUN Study) Investigators. Factors associated with prevalent abnormal anal cytology in a large cohort of HIV-infected adults in the United States. I Infect Dis 2010; 202:1567-1576.

20. Ciobotaru B, Leiman G, St John T, Hyman N, Ramundo M, Grace $\mathrm{C}$. Prevalence and risk factors for anal cytologic abnormalities and human papillomavirus infection in a rural population of HIV-infected males. Dis Colon Rectum 2007; 50:1011-1016.

21. Coutlee F, de Pokomandy A, Franco EL. Epidemiology, natural history and risk factors for anal intraepithelial neoplasia. Sex Health 2012; 9:547-555. 
22. Hillman RJ, van Leeuwen MT, Vajdic CM, McHugh L, Prestage $\mathrm{GP}$, Botes LP, et al. Prevalence and predictors of high-grade anal intraepithelial neoplasia in a community-based sample of homosexual men. Sex Health 2012; 9:574-579.

23. Melo VH, Guimaraes MD, Rocha GM, Araujo AC, Carmo RA, Grinsztejn B, et al. Prevalence and risk factors associated with anal intraepithelial neoplasia among HIV-positive men in Brazil. J Low Genit Tract Dis 2014; 18:128-135.

24. Alvarez J, de Pokomandy A, Rouleau D, Ghattas G, Vezina S, Cote $P$, et al., HIPVIRG Study Group. Episomal and integrated human papillomavirus type 16 loads and anal intraepithelial neoplasia in HIV-seropositive men. AIDS 2010; 24:2355-2363.

25. Libois A, Feoli F, Nkuize M, Delforge M, Konopnicki D, Clumeck $\mathrm{N}$, et al. Prolonged antiretroviral therapy is associated with fewer anal high-grade squamous intraepithelial lesions in HIV-positive MSM in a cross-sectional study. Sex Trans Infect 2017; 93:15-17.
26. van Aar F, Mooij SH, van der Sande MA, Speksnijder AG, Stolte IG, Meijer CJ, et al. Anal and penile high-risk human papillomavirus prevalence in HIV-negative and HIV-infected MSM. AIDS 2013; 27:2921-2931.

27. Gosens KC, De Vries HJ, Quint KD, Van Noesel C, Richel O, Van den Munckhof HA, et al. Causative human papillomavirus genotypes in anal squamous cell carcinoma: HIV-positive versus HIV-negative patients. In: IANS Scientific meeting. Atlanta, USA; 2015.

28. Richel O, Prins JM, de Vries HJ. Screening for anal cancer precursors: what is the learning curve for high-resolution anoscopy? AIDS 2014; 28:1376-1377.

29. Hillman RJ, Cuming T, Darragh T, Nathan M, Berry-Lawthorn $\mathrm{M}$, Goldstone $\mathrm{S}$, et al. 2016 IANS International Guidelines for Practice Standards in the Detection of Anal Cancer Precursors. J Low Genit Tract Dis 2016; 20:283-291. 